\title{
Shannon Information Theoretic Computation of Synonymous Codon Usage Biases in Coding Regions of Human and Mouse Genomes
}

\author{
Barry Zeeberg \\ Laboratory of Molecular Pharmacology, National Cancer Institute, National Institutes of Health, \\ Bethesda, Maryland 20892, USA
}

\begin{abstract}
Exonic GC of human mRNA reference sequences (RefSeqs), as well as $A, C, G$, and $T$ in codon position 3 are linearly correlated with genomic GC. These observations utilize information from the completed human genome sequence and a large, high-quality set of human and mouse coding sequences, and are in accord with similar determinations published by others. A Shannon Information Theoretic measure of bias in synonymous codon usage was developed. When applied to either human or mouse RefSeqs, this measure is nonlinearly correlated with genomic, exonic, and third codon position $A, C, G$, and $T$. Information values between orthologous mouse and human RefSeqs are linearly correlated: mouse $=0.092+0.55$ human. Mouse genes were consistently placed in genomic regions whose GC content was closer to $50 \%$ than was the GC content of the human ortholog. Since the (nonlinear) information versus percent GC curve has a minimum at $50 \%$ GC and monotonically increases with increasing distance from $50 \%$ GC, this phenomenon directly results in the low slope of 0.55 . This appears to be a manifestation of an evolutionary strategy for placement of genes in regions of the genome with a GC content that relates synonymous codon bias and protein folding.
\end{abstract}

[The following individuals kindly provided reagents, samples, or unpublished information as indicated in the paper: D. Church and D. Maglott.]

In a seminal study comparing human and rodent coding and noncoding sequences, Makalowski and Boguski (1998) clearly showed that synonymous substitution rates were very high, close to rates for substitutions in $5^{\prime}$ and $3^{\prime}$ untranslated regions. In sharp contrast, nonsynonymous substitution rates were about five-fold slower. This high rate of synonymous substitution might be expected to lead to a rather homogeneous distribution of synonymous codons. On the other hand, there are patterns of synonymous codon usage that are indicative of nonrandomness (for review, see Li 1997, pp. 196-202). Relying heavily on cited work by Sharp et al. (1988), Sharp and Li (1986), Ikemura (1981), Kimura (1983), and other researchers, this review shows that, in unicellular organisms, genes whose protein products are highly expressed preferentially utilized synonymous codons that corresponded to the most abundant tRNA species. In contrast, genes whose protein products are not highly expressed are more or less promiscuous in their usage of synonymous codons.

There is a comprehensive body of literature documenting the universal existence and nature of codon usage bias within and between many organisms. Professor Paul Sharp kindly shared an informal historical perspective (detailed references are given in the Results section): The earliest studies started around mid 1980's with the work of Ikemura and colleagues, and a number of other major groups continued this tradition in ongoing efforts that continue very energetically to the present time. A common focus of this work is the determination of the relationship of the nucleotide composition of codon position 3 and the local genomic nucleotide com-

E-MAIL barry@discover.nci.nih.gov; FAX (301) 402-0752. Article and publication are at http://www.genome.org/cgi/doi/10.1101/ gr.213402. position. Often this information has been used to elucidate alternative hypotheses relevant to mechanisms or pathways of genomic evolution.

I show here that an information theoretic value (in the sense of Shannon 1948) can be computed for each coding sequence based upon its usage of synonymous codons. As described here briefly and in Methods in full detail, Shannon Information for synonymous codon usage is computed as the double sum,

$$
\sum_{i=1}^{i=n_{\text {aa }}}\left(-\sum_{j=1}^{j=n_{\text {syncod }(i)}} p_{i, j} \log _{2}\left(p_{i, j}\right)\right)
$$

where $\mathrm{n}_{\mathrm{aa}}$ is the number of distinct amino acids, $\mathrm{n}_{\text {syncod(i) }}$ is the number of synonymous codons for amino acid $i$, and $p_{i, j}$ is the probability of synonymous codon $\mathrm{j}$ for amino acid $\mathrm{i}$. This information value can be used to describe the diversity of codon usage within individual coding sequences and for the genome as a whole, to compare diversity between multiple organisms, and to develop models of coding sequence evolution. For example, comparison of results for mouse and human genomes shows that there is a linear correlation of information values between orthologous mouse and human mRNA reference sequences, following the regression equation: mouse $=0.092+0.55$ human. Mouse genes were consistently placed in genomic regions whose GC content was closer to $50 \%$ than was the GC content of the human ortho$\log$. Since the (nonlinear) information versus percent GC curve has a minimum at $50 \%$ GC and monotonically increases with increasing distance from 50\% GC, this phenomenon directly results in the low slope of 0.55 . This appears to be a manifestation of an evolutionary strategy for placement 
Information Theory and Synonymous Codon Usage

of genes in regions of the genome with a GC content that relates synonymous codon bias and protein folding. This analysis indicates the potential utility of the information theoretic measure for whole genomic comparison of synonymous codon usage.

\section{RESULTS}

\section{Relationship of Percent GC in Coding Sequences of Genes and Percent GC in Human Genome}

There is a positive linear relationship between percent GC in coding sequences of genes and the percent GC in the genomic region containing the gene (Fig. 1A). These observations, which utilize a large number of high-quality sequences and information derived from the completed human genome sequence, are in accord with a vast body of results published during the last two decades (Ikemura et al. 1983; Ikemura 1985; Aota and Ikemura 1986; Bulmer 1987; Filipski et al. 1987; Mouchiroud et al. 1987; Muto and Osawa 1987; Ikemura and Aota 1988; Sharp et al. 1988; Marin et al. 1989; Ikemura et al. 1990; Ikemura and Wada 1991; Lloyd and Sharp 1993; Sharp et al. 1993; Kliman and Hey 1994; Sharp and Matassi 1994; Stenico et al. 1994; Sharp et al. 1995; Andersson and Sharp 1996; Table 7.10 of Li 1997; Lafay and Sharp 1999; Bernardi 2000a; Cruveiller et al. 2000; Lafay et al. 2000; Sueoka and Kawanishi 2000; Grocock and Sharp 2001; Knight et al. 2001). There is a small contribution to this phenomenon from codon 1 (Fig. 1B), a negligible contribution from codon 2 (Fig. 1C), and a substantial contribution from codon 3 (Fig. 1D). These relationships are similar for all four individual nucleotides (Table 1), with $\mathrm{A}$ and $\mathrm{T}$ exhibiting negative rather than positive correlations (figures not shown).
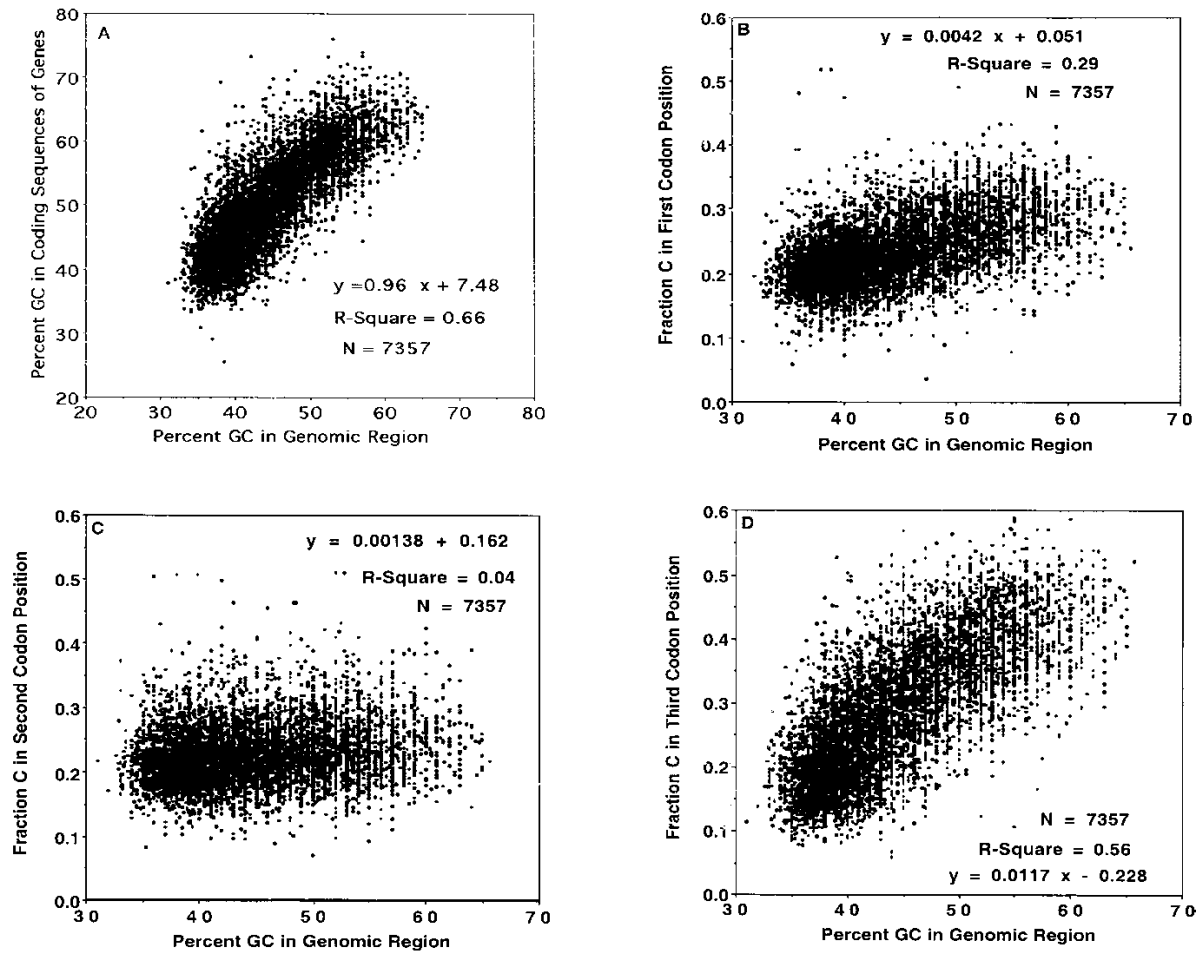

Figure 1 Relationship of $(A)$ percent $G C$ in coding sequences of genes, $(B)$ fraction $C$ in first codon position, $(C)$ fraction $C$ in second codon position, and $(D)$ fraction $C$ in third codon position to percent GC in genomic region of human sequences.
Nucleotide frequency of each codon position (position 1: $G>$ $\mathrm{A}>\mathrm{C}>\mathrm{T}$; position 2: $\mathrm{A}>\mathrm{T}>\mathrm{C}>\mathrm{G}$; position 3: $\mathrm{C}>\mathrm{G}>\mathrm{T}>$ A) is in accord with the quantitation given by Karlin and Mrázek (1996).

\section{Relationship of Human Nucleotide Content and Information (bits)}

As described here briefly and in Methods in full detail, Shannon Information for synonymous codon usage is computed as the double sum

$$
\sum_{i=1}^{i=n_{\text {aa }}}\left(-\sum_{j=1}^{j=n_{\text {syncod }(i)}} p_{i, j} \log _{2}\left(p_{i, j}\right)\right)
$$

where $\mathrm{n}_{\mathrm{aa}}$ is the number of distinct amino acids, $\mathrm{n}_{\text {syncod(i) }}$ is the number of synonymous codons for amino acid $i$, and $p_{i, j}$ is the probability of synonymous codon $j$ for amino acid $i$. There is a nonlinear relation between information in the coding region of a gene and the percent GC in the genomic region containing the gene (Fig. 2A). This relationship becomes visually clearer when the percent GC in the coding portion of the gene rather than in the genomic region is used (Fig. 2B). The shape is reminiscent of the plot of uncertainty versus probability for two variables (see, e.g., Hamming 1980; Tom Schneider, "Information Theory Primer with an Appendix on Logarithms," www.lecb.ncifcrf.gov/ toms/paper/primer): The shape in Figure $2 \mathrm{~B}$ is rescaled and inverted, since this was measured for much more than two variables, and this is a plot of information rather than uncertainty. There is no contribution to this shape from the $\mathrm{C}$ content of codon 1 (Fig. 2C) or codon 2 (Fig. 2D); the major if not only source of contribution is from codon 3 (Fig. 2E). Similar results were obtained for $\mathrm{G}$, and the mirror for $\mathrm{A}$ and $\mathrm{T}$ (not shown).

As described here briefly and in Methods in full detail, given a scatter plot of information versus the probability of one (of several) variables, the effective number of variables can be estimated as $n_{e^{-}}$ fective $=1 / p_{\min }$, where $n_{\text {effective }}$ is the effective number of variables and $p_{\min }$ is the value of $p$ at which the information achieves a minimum. For the data in Figure 2B, the minimum in the scatter plot occurs close to $50 \%$ GC; therefore, as described in Methods, $\mathrm{n}_{\text {effective }}=1 / 0.5=2$. The fit of the theoretical curve for 2 variables (Equation 5) was rather poor: the minimum for the measured data occurs at approximately 45\% GC rather than exactly at the position of the theoretical minimum at $50 \%$ GC; also, the theoretical curve tends to underestimate the bulk of the information values for $\mathrm{GC} \geq 50 \%$. On the other hand, for Figure $2 \mathrm{E}$, the minimum in the scatter plot occurs close to fraction $\mathrm{C}$ in codon position $3=0.25$; 
Table 1. Statistical Results for Linear Regression Analysis of Codon Nucleotide Composition in Relationship to Percent GC in Human Genomic Regions

\begin{tabular}{|c|c|c|c|}
\hline \multicolumn{4}{|c|}{ Slopes } \\
\hline \multirow[b]{2}{*}{ Nucleotide } & \multicolumn{3}{|c|}{ Codon position } \\
\hline & 1 & 2 & 3 \\
\hline A & -0.0039 & -0.00282 & -0.0102 \\
\hline C & 0.0042 & 0.00138 & 0.0117 \\
\hline G & 0.00162 & 0.00195 & 0.0084 \\
\hline $\mathrm{T}$ & -0.00192 & -0.00051 & -.0100 \\
\hline \multicolumn{4}{|c|}{ Intercepts } \\
\hline & \multicolumn{3}{|c|}{ Codon position } \\
\hline Nucleotide & 1 & 2 & 3 \\
\hline A & 0.45 & 0.45 & 0.66 \\
\hline $\mathrm{C}$ & 0.051 & 0.162 & -0.228 \\
\hline G & 0.243 & 0.100 & -0.090 \\
\hline $\mathrm{T}$ & 0.258 & 0.291 & 0.66 \\
\hline \multicolumn{4}{|c|}{ R-Square values } \\
\hline & \multicolumn{3}{|c|}{ Codon position } \\
\hline Nucleotide & 1 & 2 & 3 \\
\hline A & 0.29 & 0.10 & 0.60 \\
\hline C & 0.29 & 0.04 & 0.56 \\
\hline G & 0.06 & 0.10 & 0.56 \\
\hline $\mathrm{T}$ & 0.11 & 0.004 & 0.62 \\
\hline
\end{tabular}

therefore, $\mathrm{n}_{\text {effective }}=1 / 0.25=4$. The fit to the theoretical curve for four variables (Equation 6) appears to be better than that in Figure 2B: the minima for both the measured data and the theoretical curve coincide at 0.25 , and the theoretical curve comes close to running through the middle of the measured data. As analyzed in detail in the Discussion, the measured data in Figure 2C,D deviate markedly from the theoretical curve.

\section{Identification of "Rare" Codon Usage and Relationship to Information Value}

For reference, the nucleotide triplets for each synonymous codon for each amino acid are numbered and tabulated (Table 2 ). The positions of numerical values (codon frequencies) in Tables 3-7 correspond to the positions in reference Table 2 . The results in Table 3 are in excellent agreement with those in the gold standard tabulation (Nakamura 1998; www.kazusa.or.jp/codon/cgi-bin/showcodon.cgi?species=Homo+ sapiens+[gbpri]). The codon frequencies for the pooled 10,862 human RefSeqs were normalized for the pooled total (Table 3) and for each individual amino acid (Table 4). Visual inspection of Table 4 indicates that there are no amino acids that have extremely rarely used codons which might be expected to be involved in translational control. Moderately rarely used codons were identified by dividing each codon frequency in Table 4 by the maximum frequency for the corresponding amino acid (Table 5). The rank order of the pooled and sorted rarely used codons is indicated in parentheses for values $\leq 0.50$. The most rarely used codon is codon 3 of leucine (CTA) with a ratio of 0.181 relative to codon 4 of leucine (CTG).
Analyses were performed for two arbitrarily selected RefSeqs, NM_000065 (Homo sapiens complement component 6; Table 6) with a low information value (0.00 bits), and NM_002246 (Homo sapiens potassium channel, subfamily K, member 3; Table 7), with a high information value (16.12 bits). These analyses are described in detail in the Discussion.

The fraction of frequent codons is inversely linearly related to the fraction of rare codons (Fig. 3A). However, this observation may not entirely reflect a biologically relevant relationship, since a sequence that contains, for example, a frequent codon fraction of 0.80 can contain at most a rare codon fraction of 0.20 , so that at least a portion of the inverse relationship may be attributable to a mathematical constraint rather than to biological selection.

The information value exhibits a nonlinear relationship to the fraction of rare codons (Fig. 3B), reminiscent of the relationship of the information value to $\mathrm{A}$ or $\mathrm{T}$ in codon position 3 (not shown). The information value exhibits a nonlinear relationship to the fraction of frequent codons (Fig. $3 \mathrm{C})$, reminiscent of the relationship of the information value to $\mathrm{C}$ (Fig. 2E) or $\mathrm{G}$ (not shown) in codon position 3. The details of the individual and the large-scale information values analyses are presented in the Discussion.

\section{Comparison with Mouse}

The relationship of mouse nucleotide content and information values (Fig. 4A-D) was very similar to those for human (Fig. 2B-E). The comments above regarding the theoretical curve fits for human apply similarly to mouse, and are analyzed in detail in the Discussion.

Linear regression analysis for 2902 mouse versus human orthologous RefSeq pairs (D. Maglott, pers. comm.) showed linear correlations for percent GC in coding sequences of genes (Fig. 5A). This was also true for the composition of all four nucleotides at all three codon positions (Table 8; Fig. 5B); similar analyses have been published by Mouchiroud and Bernardi (1993) and Bernardi (2000b) for orthologous pairs of genes in several species.

The mouse and human orthologs also exhibited a linear relationship for both fraction of rare (Fig. 5C) and fraction of frequent (Fig. 5D) codons. The implications of this observation are analyzed in detail in the Discussion.

The information values for orthologous mouse and human RefSeqs were linearly correlated (Fig. 5E), following the regression equation: mouse $=0.092+0.55$ human. This relationship could be modeled quite closely (Fig. 5F) by substituting the fraction $\mathrm{C}$ in codon position 3 (i.e., each point in Fig. 5B) for " $p$ " in Equation 6, for both mouse and human. This allowed a transformation from "fraction C orthologous pairs" (fraction $\mathrm{C}$ in third human codon position, fraction $\mathrm{C}$ in third mouse codon position) to "model information orthologous pairs" (human information computed from model, mouse information computed from model). The regression equation for the model fit (i.e., the scatter plot of "model information orthologous pairs;" Fig. 5F) was similar to that for the measured data (Fig. 5E): mouse $=0.21+0.48$ human.

\section{DISCUSSION}

\section{Relationship of Percent GC in Coding Sequences} of Genes and Percent GC in Human Genome

The positive correlation between percent GC in coding sequences of genes and the percent GC in the genomic region 

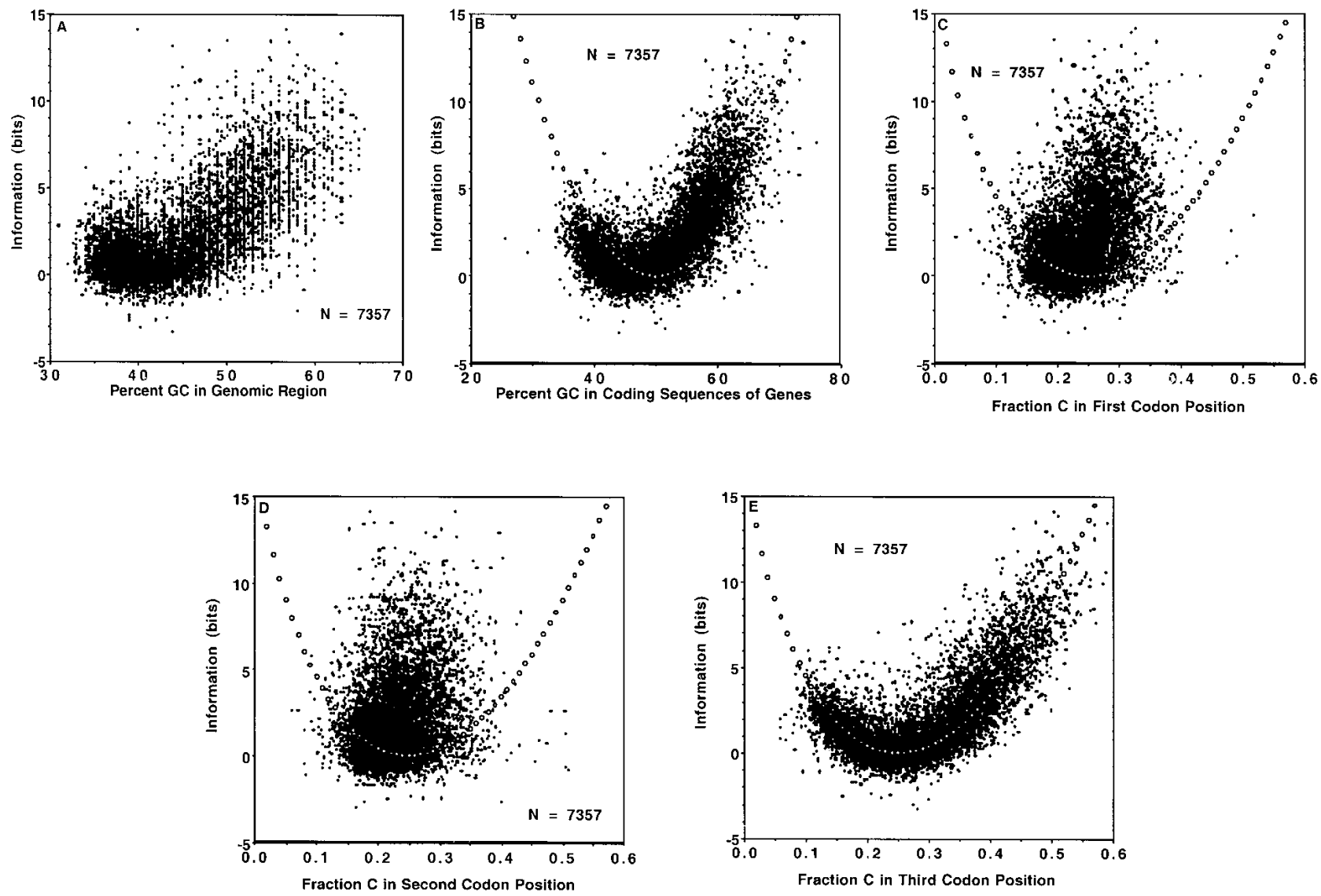

Figure 2 Relationship of information (bits) and percent GC, in (A) genomic regions of human sequences; and relationship of information (bits) and $C$ content in $(B)$ coding sequences of human genes, $(C)$ first codon position for human sequences, $(D)$ second codon position for human sequences; and $(E)$ third codon position for human sequences. Open circles indicate theoretical fit to the information function.

containing the gene (Fig. 1A) is somewhat surprising: the number of nucleotides in coding sequences is generally a small fraction of the number of nucleotides in the genomic region spanning the gene, since in human the introns are much longer than the exons, and the $20 \mathrm{~kb}$ window used in computing the genomic percent GC adds even more nonexonic sequence at the termini of the spanning genomic region. Thus, it is highly unlikely that the genomic percent GC is numerically a consequence of the coding sequence percent GC. The same argument would apply even more strongly to the correlation with fraction $\mathrm{C}$ (Fig. 1D; Table 1) or G in the third codon position. There is simply not enough $\mathrm{G}$ or $\mathrm{C}$ in one codon position to substantially affect the genomic GC content. By logical elimination, it is likely that the same factor(s) from which genomic regions of different characteristic percent GC arose also caused the correlation with the third codon position. The second codon position was not subject to this factor (Fig. 1C), since this position is instrumental in determining the identity of the amino acid, so that strong functional constraints would completely override the tendency toward GC bias. These same constraints are apparently present, but not as strongly, in the first codon position (Fig. 1B). In fact, this position has a minor characteristic of a wobble position in that there are synonymous substitutions in codon position 1 for leucine and arginine (see Methods). Although there are no such synonymous substitutions in codon position 2 for leucine and arginine, it should be mentioned that the combination of positions 1 and 2 could be regarded as a wobble position for serine (TCT/AGT and TCC/ AGC), but this type of "concerted" synonymous substitution would have no effect on the A, C, G, or T content of any of the three codon positions.

\section{Relationship of Human Nucleotide Content and Information (bits)}

A fundamental observation is that the information value is nonlinearly correlated with GC content (and, since AT $=1$ GC, also with AT content) in the genome (Fig. 2A) and in the coding sequence (Fig. 2B), and with the A, C, G, or T content in the third codon position (Fig. 2E). This observation can be understood by considering the GC content in the coding sequence (Fig. 2B): as percent GC or AT approaches the maximal value (that is, as the percent GC or AT increases or decreases from 50\%), there are fewer choices of synonymous codons available, so that the information tends towards a relatively high value. Because of the linear correlation of GC content in the coding sequence with GC content in the genomic region (Fig. 1A) and with A, C, G, and T in codon position 3 (Fig. 1D), these also "inherit" the nonlinear relation between information value and GC content in the coding sequence.

This phenomenon is the biological analogy of the proba- 
Table 2. Positional Correspondence of Condons for Subsequent Tables

\begin{tabular}{|c|c|c|c|c|c|c|}
\hline \multirow{2}{*}{$\begin{array}{l}\text { Amino } \\
\text { acid }\end{array}$} & \multicolumn{6}{|c|}{ Codon number } \\
\hline & 1 & 2 & 3 & 4 & 5 & 6 \\
\hline PHE & TTT & TTC & & & & \\
\hline LEU & $\mathrm{CTT}$ & CTC & CTA & CTG & TTA & TTG \\
\hline ILE & ATT & ATC & ATA & & & \\
\hline MET & ATG & & & & & \\
\hline VAL & GTT & GTC & GTA & GTG & & \\
\hline SER & TCT & TCC & TCA & TCG & AGT & AGC \\
\hline PRO & $\mathrm{CCT}$ & $\mathrm{CCC}$ & $\mathrm{CCA}$ & CCG & & \\
\hline THR & $\mathrm{ACT}$ & $\mathrm{ACC}$ & $\mathrm{ACA}$ & ACG & & \\
\hline ALA & GCT & GCC & GCA & GCG & & \\
\hline TYR & TAT & TAC & & & & \\
\hline HIS & $\mathrm{CAT}$ & CAC & & & & \\
\hline GLN & CAA & CAG & & & & \\
\hline ASN & $\mathrm{AAT}$ & AAC & & & & \\
\hline LYS & $\mathrm{AAA}$ & AAG & & & & \\
\hline ASP & GAT & GAC & & & & \\
\hline GLU & GAA & GAG & & & & \\
\hline CYS & TGT & TGC & & & & \\
\hline TRP & TGG & & & & & \\
\hline ARG & CGT & CGC & CGA & CGG & AGA & AGG \\
\hline GLY & GGT & GGC & GGA & GGG & & \\
\hline
\end{tabular}

bilistic argument leading to the nonlinear relationship in the plot of uncertainty versus probability for two variables (see, e.g., Hamming 1980; Tom Schneider, "Information Theory Primer with an Appendix on Logarithms," www.lecb.ncifcrf. gov/ toms/paper/primer). As the probability deviates either positively or negatively from $50 \%$, the information approaches its maximum value. The biological observation is all the more important in that the nonlinear relationship is present for codon position 3 (Fig. 2E), but not for codon positions 1 (Fig. 2C) or 2 (Fig. 2D). The high quality of the theoretical fit for codon position 3 (Fig. 2E) indicates that the

Table 3. Pooled Synonymous Codon Frequencies for 10862 Human RefSeqs Normalized by Pooled Totals

\begin{tabular}{lcccccc}
\hline & \multicolumn{7}{c}{ Codon number } \\
\cline { 2 - 7 } $\begin{array}{l}\text { Amino } \\
\text { acid }\end{array}$ & $\mathbf{1}$ & $\mathbf{2}$ & $\mathbf{3}$ & $\mathbf{4}$ & $\mathbf{5}$ & 6 \\
\hline PHE & 0.017 & 0.020 & & & & \\
LEU & 0.013 & 0.019 & 0.007 & 0.039 & 0.008 & 0.013 \\
ILE & 0.016 & 0.021 & 0.007 & & & \\
MET & 0.022 & & & & & \\
VAL & 0.011 & 0.014 & 0.007 & 0.028 & & \\
SER & 0.015 & 0.017 & 0.012 & 0.004 & 0.012 & 0.019 \\
PRO & 0.018 & 0.020 & 0.017 & 0.007 & & \\
THR & 0.013 & 0.019 & 0.015 & 0.006 & & \\
ALA & 0.019 & 0.028 & 0.016 & 0.007 & & \\
TYR & 0.012 & 0.015 & & & & \\
HIS & 0.011 & 0.015 & & & & \\
GLN & 0.012 & 0.034 & & & & \\
ASN & 0.018 & 0.020 & & & & \\
LYS & 0.025 & 0.033 & & & & \\
ASP & 0.023 & 0.026 & & & & \\
GLU & 0.030 & 0.040 & & & & \\
CYS & 0.010 & 0.012 & & & & \\
TRP & 0.012 & & & & & \\
ARG & 0.005 & 0.010 & 0.006 & 0.011 & 0.012 & 0.011 \\
GLY & 0.011 & 0.023 & 0.017 & 0.016 & & \\
\hline
\end{tabular}

Table 4. Pooled Synonymous Codon Frequencies for 10862 Human RefSeqs Normalized by Amino Acid Totals

\begin{tabular}{lcccccc}
\hline & \multicolumn{5}{c}{ Codon number } \\
Amino & \multicolumn{7}{c}{ acid } & 1 & 2 & 3 & 4 & 5 & 6 \\
\cline { 2 - 6 } & & & & & \\
PHE & 0.465 & 0.535 & & & & \\
LEU & 0.132 & 0.191 & 0.072 & 0.397 & 0.078 & 0.130 \\
ILE & 0.364 & 0.473 & 0.162 & & & \\
MET & 1.000 & & & & & \\
VAL & 0.182 & 0.236 & 0.118 & 0.464 & & \\
SER & 0.185 & 0.215 & 0.149 & 0.056 & 0.154 & 0.240 \\
PRO & 0.288 & 0.321 & 0.278 & 0.113 & & \\
THR & 0.247 & 0.353 & 0.283 & 0.117 & & \\
ALA & 0.266 & 0.399 & 0.230 & 0.104 & & \\
TYR & 0.445 & 0.555 & & & & \\
HIS & 0.421 & 0.579 & & & & \\
GLN & 0.265 & 0.735 & & & & \\
ASN & 0.472 & 0.528 & & & & \\
LYS & 0.432 & 0.568 & & & & \\
ASP & 0.471 & 0.529 & & & & \\
GLU & 0.431 & 0.569 & & & & \\
CYS & 0.456 & 0.544 & & & & \\
TRP & 1.000 & & & & & \\
ARG & 0.084 & 0.188 & 0.014 & 0.206 & 0.208 & \\
GLY & 0.165 & 0.340 & 0.253 & 0.242 & & \\
\hline & & & & & & \\
\hline
\end{tabular}

choice of codon position 3 and therefore of synonymous codons is under, at most, quite modest biological constraint, and is governed primarily by probabilistic and statistical considerations. On the other hand, the deviation from the theoretical fit for codon positions 1 and 2 (Fig. 2C,D) indicates that the choice of codon positions 1 and 2 are under severe biological constraint (i.e., the identity of the amino acid) that causes a marked deviation from what would be expected if based solely upon probabilistic and statistical considerations. If the choice of synonymous codons were under biological constraint, then Figure 2E would look like Figure 2C,D, and the data in Figure $2 \mathrm{E}$ would not correspond to the theoretical fit. The lower quality of the theoretical fit for percent GC in coding sequences (Fig. 2B) relative to the theoretical fit for fraction $\mathrm{C}$ in codon position 3 (Fig. 2E) is easily explained by the fact that the former is "contaminated" by including codon positions 1 and 2, which exhibit a very poor fit (Fig. 2B,C).

The conclusion that synonymous codon choice is under, at most, quite modest biological constraint appears to be inconsistent with the classical findings in unicellular organisms that genes whose protein products are highly expressed are under a strong biological constraint, in that they preferentially use the most frequent codons (see above). This general conclusion is supported by examination of certain specific classes of proteins that are considered to be highly expressed. For example, within the set of human RefSeqs studied here, 106 were identified as coding for ribosomal proteins. The mean \pm standard deviation for the information value, fraction of rare codons, and fraction of frequent codons are 2.60 $\pm 2.35,0.13 \pm 0.04$, and $0.43 \pm 0.11$, respectively; these values are very similar to those for various sets of housekeeping genes (not shown). The corresponding values for the complete set of human RefSeqs are $2.63 \pm 2.81,0.14 \pm 0.03$, and $0.44 \pm 0.12$. Thus the highly expressed ribosomal proteins and housekeeping gene products are statistically similar to the complete set of genes. When the highly expressed ribosomal proteins and housekeeping gene products are removed from 
Table 5. Ratio to Maximum and Rank Ordering of Rare Codons Computed from Pooled Synonymous Codon Frequencies for 10,862 Human RefSeqs

\begin{tabular}{|c|c|c|c|c|c|c|}
\hline \multirow{2}{*}{$\begin{array}{l}\text { Amino } \\
\text { acid }\end{array}$} & \multicolumn{6}{|c|}{ Codon number } \\
\hline & 1 & 2 & 3 & 4 & 5 & 6 \\
\hline PHE & 0.869 & 1.000 & & & & \\
\hline LEU & $0.332(8)$ & $0.481(14)$ & $0.181(1)$ & 1.000 & $0.196(2)$ & $0.327(6)$ \\
\hline ILE & 0.770 & 1.000 & $0.342(9)$ & & & \\
\hline MET & 1.000 & & & & & \\
\hline VAL & $0.392(12)$ & 0.509 & $0.254(4)$ & 1.000 & & \\
\hline SER & 0.771 & 0.896 & 0.621 & $0.233(3)$ & 0.642 & 1.000 \\
\hline PRO & 0.897 & 1.000 & 0.866 & $0.352(10)$ & & \\
\hline THR & 0.700 & 1.000 & 0.802 & $0.331(7)$ & & \\
\hline ALA & 0.667 & 1.000 & 0.576 & $0.261(5)$ & & \\
\hline TYR & 0.802 & 1.000 & & & & \\
\hline HIS & 0.727 & 1.000 & & & & \\
\hline GLN & $0.361(11)$ & 1.000 & & & & \\
\hline ASN & 0.894 & 1.000 & & & & \\
\hline LYS & 0.761 & 1.000 & & & & \\
\hline ASP & 0.890 & 1.000 & & & & \\
\hline GLU & 0.757 & 1.000 & & & & \\
\hline CYS & 0.838 & 1.000 & & & & \\
\hline TRP & 1.000 & & & & & \\
\hline ARG & $0.404(13)$ & 0.904 & 0.548 & 0.990 & 1.000 & 0.966 \\
\hline GLY & $0.485(15)$ & 1.000 & 0.744 & 0.712 & & \\
\hline
\end{tabular}

2 and 3 , but in which only the genes for highly expressed proteins are included, are visually identical to the corresponding plots in Figures 2 and 3. Thus, there is no evidence that these genes are more highly constrained than the general population of genes.

\section{Identification of "Rare" Codon Usage and Relationship to Information Value}

An obvious hypothesis for explaining the existence of some sequences with a high information value is that these sequences use a preponderance of "rare" codons for translational control. However, "rare" codons are in fact rare or nonexistent (Table 5). The rarest codon is codon 3 of leucine, and this is used at $18.1 \%$ the frequency of the maximally used codon 4 of leucine.

It is notable that the codon with the maximum usage for each

the complete set, the resulting set, which is specifically diminished in genes for highly expressed proteins, has corresponding values of $2.42 \pm 2.63,0.14 \pm 0.04$, and $0.42 \pm 0.12$. Thus the highly expressed ribosomal proteins and housekeeping gene products are statistically similar to the set of genes which is specifically diminished in genes for highly expressed proteins. In summary, in contrast to the unicellular organisms, human does not appear to preferentially utilize frequent codons for genes with highly expressed protein products.

In addition, plots that are analogous to those in Figures

Table 6. Synonymous Codon Frequencies for RefSeq NM_000065 Relative to Pooled Synonymous Codon Frequenceies for 10862 Human RefSeqs

\begin{tabular}{lcccccc}
\hline & \multicolumn{6}{c}{ Codon number } \\
\cline { 2 - 7 } amino & $\mathbf{1}$ & $\mathbf{2}$ & $\mathbf{3}$ & $\mathbf{4}$ & $\mathbf{5}$ & $\mathbf{6}$ \\
\hline PHE & 1.435 & 0.623 & & & & \\
LEU & 1.110 & 0.693 & 1.430 & 0.630 & 1.893 & 1.696 \\
ILE & 1.267 & 0.704 & 1.264 & & & \\
MET & 1.000 & & & & & \\
VAL & 1.276 & 1.183 & 1.771 & 0.602 & & \\
SER & 1.365 & 0.841 & 1.775 & 0.215 & 1.094 & 0.502 \\
PRO & 0.969 & 0.870 & 1.506 & 0.205 & & \\
THR & 1.373 & 0.695 & 1.335 & 0.323 & & \\
ALA & 1.222 & 0.876 & 1.412 & 0.000 & & \\
TYR & 1.083 & 0.934 & & & & \\
HIS & 1.627 & 0.545 & & & & \\
GLN & 1.643 & 0.769 & & & & \\
ASN & 1.186 & 0.833 & & & & \\
LYS & 1.369 & 0.720 & & & & \\
ASP & 1.133 & 0.881 & & & & \\
GLU & 1.516 & 0.609 & & & & \\
CYS & 1.199 & 0.833 & & & & \\
TRP & 1.000 & & & & \\
ARG & 0.509 & 0.681 & 1.117 & 0.414 & 1.843 & 1.163 \\
GLY & 0.917 & 0.624 & 1.618 & 0.940 & & \\
\hline
\end{tabular}

amino acid usually has C (or, less often, G) in codon position 3 . It is also notable that the four four-codon families which have $\mathrm{C}$ in codon position 2 (i.e., serine, proline, threonine, and alanine, if we momentarily ignore the two "extra" serine codons that contain $G$ in codon position 2) all contain a codon (codon 4 in all four cases) that is identified as a "rarely used" codon. It is perhaps somewhat surprising that these four codons all contain $\mathrm{G}$ in position 3, given the comment above concerning codons with maximum usage.

Two RefSeqs were arbitrarily chosen for manual exami-

Table 7. Synonymous Codon Frequencies for RefSeq NM 002246 Relative to Pooled Synonymous Codon Frequencies for 10862 Human RefSeqs

\begin{tabular}{lcccccc}
\hline & \multicolumn{7}{c}{ Codon number } \\
\cline { 2 - 7 } $\begin{array}{l}\text { Amino } \\
\text { acid }\end{array}$ & $\mathbf{1}$ & $\mathbf{2}$ & $\mathbf{3}$ & $\mathbf{4}$ & $\mathbf{5}$ & $\mathbf{6}$ \\
\hline PHE & 0.000 & 1.868 & & & & \\
LEU & 0.180 & 1.620 & 0.000 & 1.621 & 0.000 & 0.183 \\
ILE & 0.000 & 2.113 & 0.000 & & & \\
MET & 1.000 & & & & & \\
VAL & 0.000 & 1.237 & 0.000 & 1.528 & & \\
SER & 0.000 & 1.692 & 0.203 & 3.785 & 0.000 & 1.640 \\
PRO & 0.000 & 1.247 & 0.360 & 4.405 & & \\
THR & 0.144 & 1.315 & 0.000 & 4.278 & & \\
ALA & 0.000 & 1.325 & 0.256 & 3.942 & & \\
TYR & 0.000 & 1.801 & & & & \\
HIS & 0.000 & 1.726 & & & & \\
GLN & 0.000 & 1.360 & & & & \\
ASN & 0.000 & 1.894 & & & & \\
LYS & 0.000 & 1.760 & & & & \\
ASP & 0.193 & 1.717 & & & & \\
GLU & 0.000 & 1.757 & & & & \\
CYS & 0.000 & 1.839 & & & & \\
TRP & 1.000 & & & & \\
ARG & 0.000 & 3.494 & 0.302 & 1.174 & 0.000 & 0.343 \\
GLY & 0.319 & 2.167 & 0.208 & 0.653 & & \\
\hline
\end{tabular}





Figure $3(A)$ Relationship of frequent codons and rare codons, $(B)$ relationship of information (bits) and rare codons, and $(C)$ relationship of information (bits) and frequent codons for human sequences.

\section{Comparison with Mouse}

The relationship of mouse nucleotide content and information values (Fig. 4A-D) was very similar to that for human (Fig. 2B-E), and the comments for human sequences concerning each codon position equally apply to mouse. Again, the comments regarding the human theoretical fits equally apply to mouse.

Linear regression analysis for 2902 mouse versus human orthologous RefSeq pairs showed linear correlations for the composition of all four nucleotides at all three codon positions (Table 8). As expected, the slopes are closest to unity and the R-Square values are generally highest for codon position 2 , since this position is the most constrained by the functional requirement for the identity of the amino acid. The opposite is true for codon position 3 , which has the most flexibility in terms of synonymous substitutions notwithstanding the fact that codon position 3 has the most flexibility in terms of synonymous substitution, the R-Square values are still very high.

Despite the rather high rate of synonymous divergence be-

nation. NM_000065 (information $=0.00$ bits) and NM_002246 (information $=16.12$ bits) are representatives of coding sequences with low and high information, respectively. NM_000065 (Table 6; computed as the ratios of the codon frequencies for the sequence to the corresponding entries in Table 3) exhibits a modest amount of deviation from the pooled pattern, with the exception of a low usage of the four rare-usage codons mentioned above (codon 4 for serine, proline, threonine, and alanine). However, NM_000065 fully utilizes other rare-usage codons, such as codons 3,5 , and 6 of leucine.

In stark contrast, NM_002246 (Table 7) is extremely reluctant to use codons 1,3 , or 5 of any amino acid, and strongly overutilizes the rare-usage codon 4 for serine, proline, threonine, and alanine. However, NM_002246 frequently utilizes codon 2 of all amino acids, which is almost invariably the codon with maximum usage (Table 6) for each amino acid.

The relationship between information values and the fraction of frequent codons within a sequence (Fig. 3C) is reminiscent of the relationship between information values and fraction $\mathrm{C}$ in codon position 3 (Fig. 2E). In fact, most of the frequent codons in multicodon amino acids have $\mathrm{C}$ in codon position 3 (Table 5), so this similarity is expected. As a consequence of the inverse linear correlation between the fraction of rare and the fraction of frequent codons (Fig. 3A), the mirror-image type behavior is expected for the fraction of rare codons (Fig. 3B). tween rodent and human (Makalowski and Boguski 1998), there is a linear correlation in the information values for human/mouse orthologs (Fig. 5E), following the regression equation: mouse $=0.092+0.55$ human. The fact that the slope is substantially less than unity is the direct result of the relationship of fraction $\mathrm{C}$ in codon position 3 for mouse versus human (Fig. 5B): when the theoretical model for information versus $\mathrm{p}$ (Equation 6) is applied to the data in Figure 5B, the resulting model scatter plot (Fig. $5 \mathrm{~F}$ ) is similar to the measured scatter plot (Fig. 5E).

In fact, it is easy to see that the relationship between mouse and human information (Fig. 5E) is a direct consequence of a special property of the relationship of fraction $\mathrm{C}$ in mouse versus human (Fig. 5B): when fraction $C$ in human is approximately 0.25 , there is a transition relative to the line of identity (white line through the data points) such that fraction $\mathrm{C}$ in mouse exceeds that in human below this transition point, and such that fraction $\mathrm{C}$ in human exceeds that in mouse above this transition point. Thus, for orthologous pairs, the distance from 0.25 of fraction $C$ in humans is remapped in mouse such that the distance from 0.25 is smaller in mouse than in human. The nonlinear relationship governing the data in Fig. 2E indicates that this remapping leads to a smaller information value in mouse, since the information value is at a minimum at 0.25 and it increases as fraction $C$ moves away, either positively or negatively, from 0.25 . It is indeed remarkable that the transition in the fraction $C$ data occurs almost exactly where the information minimum occurs. 


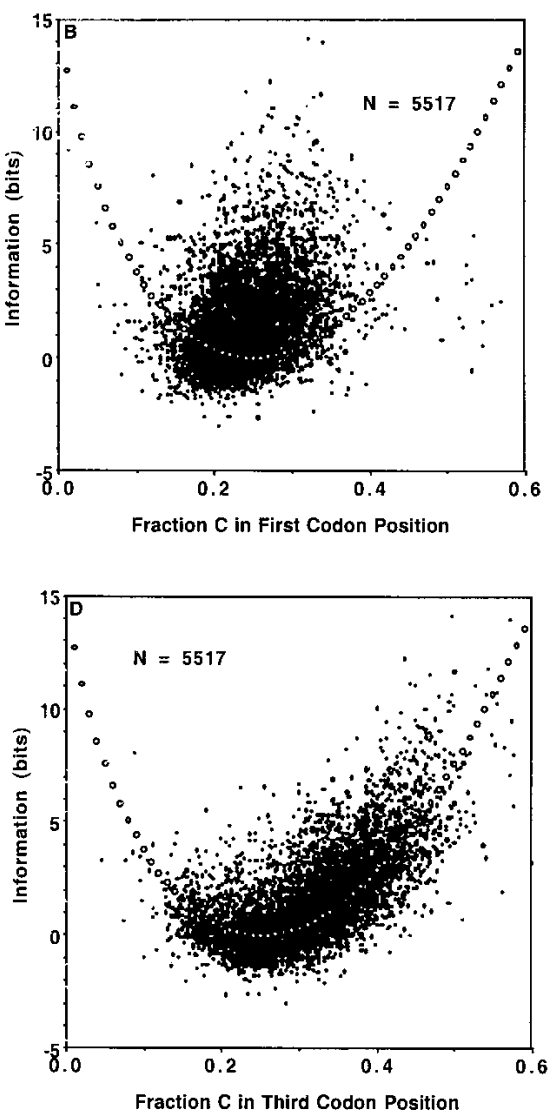

Figure 4 Relationship of information (bits) and percent $\mathrm{GC}$, in $(A)$ coding sequences of mouse genes and relationship of information (bits) and $C$ content in (B) first codon position for mouse sequences, $(C)$ second codon position for mouse sequences, and $(D)$ third codon position for mouse sequences. Open circles indicate theoretical fit to the information function.

This effect does not occur as an isolated phenomenon. The fraction $\mathrm{C}$ in codon position 3 is strongly dependent upon percent GC in the coding sequence (Fig. 1D). It is no surprise that the same phenomenon occurs for percent GC in coding sequences at approximately 50\% GC in human (Fig. $5 \mathrm{~A})$. The discussion relating to fraction $\mathrm{C}$ in the preceding paragraph also applies here in relationship to percent GC in coding sequences. Because percent GC in coding sequences is, in turn, strongly dependent upon percent GC in genomic region (Fig. 1A), it is clear that there is a chain linking (1) placement of a gene within a genomic region of a given GC composition, (2) GC composition of the coding sequence, (3) fraction $\mathrm{C}$ in codon position 3 , and (4) information value.

This same effect is present in the relationship of fraction of frequent (Fig. 5D) but not fraction of rare (Fig. 5C) codons. Again, the transition for frequent codons occurs at approximately the same position as does the information value minimum (Fig. 3C). The effect is to remap the human fraction of frequent codons to a mouse value that is closer to that for the information minimum. In essence, mouse does not utilize the full dynamic range that human does in modulating frequent codon usage. It is not clear at this time whether there is a significance to the absence of this effect for fraction of rare codons.

These observations suggest that, for a given organism, evolution may place a given gene in a region of a particular
GC content so as to adjust the information to the appropriate value for that gene in that organism. The concept of "appropriate value" for information is based upon the idea that the information value of a protein-coding gene is largely determined by the GC content of the genomic region in which the gene is embedded, perhaps through the intermediacy of the genomic GC content upon the composition of codon position 3. This is important because other important properties of the gene may themselves be determined through the intermediacy of the composition of the information value. A specific example of this is the composition of frequent and rare codons. However, the role of the information value may well extend to determining other properties of the gene that are important for adequate functionality. Placement of the gene in this manner is a strategy that utilizes the already existing mechanism for isochore maintenance to robustly maintain the appropriate information value, and consequently to maintain the appropriate composition of rare and frequent codons (Fig. $3 \mathrm{~B}, \mathrm{C})$. This strategy is particularly economical, since it utilizes the already existing mechanism for isochore maintenance; it is particularly robust, since it requires only that the information values follow the theoretical curve (Figs. 2E and 4D), which is the expected "maximum entropy" behavior for an ensemble that is not subject to external constraints. In contrast, maintenance of the identity of the amino acid requires costly external constraints, since codon positions 1 and 2 deviate substantially from the theoretical curve (Figs. 2C,D and $4 \mathrm{~B}, \mathrm{C})$.

Postulating an evolutionary strategy of gene placement requires a slight modification of the conclusion that "the choice of codon position 3 and therefore of synonymous codons is under, at most, quite modest biological constraint, and is governed primarily by probabilistic and statistical considerations." While it is still true that the choice of synonymous codons is governed primarily by probabilistic and statistical considerations, it is not quite true that the choice of synonymous codons is under, at most, quite modest biological constraint. There is a subtle constraint, namely that the gene was placed in a genomic region of a particular GC content.

Furthermore, is there any significance to controlling the fraction of rare and of frequent codons via the relationship with information value (Fig. 3B,C)? Unlike unicellular organisms, it does not appear that translation is regulated in human by synonymous codon choice. On the other hand, in mammals, but not in E. coli, there is a relationship between syn- 



Figure 5 Relationship of $(A)$ percent GC in coding sequences (white line through data points indicates identity: $G C$ in mouse $=$ GC in human), $(B)$ fraction $C$ in third codon position (white line through data points indicates identity: fraction $C$ in codon position 3 in mouse $=$ fraction $C$ in codon position 3 in human), (C) fraction of rare codons, $(D)$ fraction of frequent codons, $(E)$ information (bits), and $(F)$ model-computed information (bits) in orthologous mouse and human sequences.

onymous codon choice and protein structure (Tao and Dafu 1998). Presumably, this indicates that in mammals, folding and structure of the nascent polypeptide in the ribosome complex are under the control of synonymous codon choice. This can explain why human does not exhibit the correlation between protein expression level and synonymous codon choice: synonymous codon choice is utilized in human for control of protein folding rather than for regulation of translation.

These results and analyses indicate the potential utility of the information theoretic measure for interpretation of whole genomic comparison of synonymous codon usage, and for the inference of evolutionary mechanisms.

\section{METHODS}

\section{Coding Sequences}

Reference mRNA sequences (RefSeqs; Pruitt et al. 2000; Pruitt and Maglott 2001) for human and mouse were downloaded from www.ncbi.nlm.nih.gov on March 2, 2001 and March 16, 2001 , respectively. These files contained 10,995 and 5623 sequences, respectively. The sequences in these files were converted to FASTA format and were truncated to include only protein-coding region. After removal of sequences that contained symbols other than A, C, G, or T, there were 10,862 and 5517 sequences, respectively, that were used for subsequent analyses.

\section{Human Genomic GC Content}

The GC content of the genomic region (NCBI public genome build 22 in effect March, 2001) spanning each RefSeq was determined from Spidey (Wheelan, Church, and Ostell 2001) alignments. Each contig containing a Spidey hit was partitioned into adjacent $20 \mathrm{~kb}$ segments, and the GC content was computed for each segment. Finally, the average GC content for the adjacent genomic segments spanning each RefSeq was computed.

\section{Computation of Fraction $\mathrm{A}, \mathrm{C}, \mathrm{G}$, and $\mathrm{T}$ in Codon Positions 1, 2, and 3}

For each coding sequence, the fraction $\mathrm{F}_{\mathrm{n}, \mathrm{p}}$ of nucleotide $n \in\{A, C, G, T\}$ in codon position $p \in\{1,2,3\}$ was computed as

$$
\begin{aligned}
\mathrm{F}_{\mathrm{n}, \mathrm{p}}= & (\text { occurrences of } \mathrm{n} \text { in position } \mathrm{p}) / \\
& (\text { total number of nucleotides in position } \mathrm{p})
\end{aligned}
$$

\section{Computation of Pooled Codon Frequencies and Identification of "Rare" Codons}

For the pooled coding sequences from a given organism (Tables 3-5 for human), the number of occurrences of each codon was tabulated. The synonymous codon frequencies were computed by dividing the number of occurrences of each synonymous codon either by the pooled total over all the amino acids or, for a given amino acid, by the sum of the number of occurrences of synonymous codons for that amino 
Table 8. Linear Regression Results for Mouse versus Human Orthologous RefSeq Codon Nucleotide Composition

\begin{tabular}{|c|c|c|c|}
\hline \multicolumn{4}{|c|}{ Slopes } \\
\hline \multirow[b]{2}{*}{ Nucleotide } & \multicolumn{3}{|c|}{ Codon position } \\
\hline & 1 & 2 & 3 \\
\hline A & 0.88 & 0.95 & 0.67 \\
\hline C & 0.87 & 0.95 & 0.68 \\
\hline G & 0.92 & 0.91 & 0.65 \\
\hline $\mathrm{T}$ & 0.92 & 0.97 & 0.61 \\
\hline \multicolumn{4}{|c|}{ Intercepts } \\
\hline & \multicolumn{3}{|c|}{ Codon position } \\
\hline Nucleotide & 1 & 2 & 3 \\
\hline A & 0.011 & 0.005 & 0.019 \\
\hline C & 0.010 & 0.004 & 0.033 \\
\hline G & 0.008 & 0.006 & 0.034 \\
\hline $\mathrm{T}$ & 0.005 & 0.002 & 0.028 \\
\hline \multicolumn{4}{|c|}{ R-Square values } \\
\hline & \multicolumn{3}{|c|}{ Codon position } \\
\hline Nucleotide & 1 & 2 & 3 \\
\hline A & 0.92 & 0.94 & 0.82 \\
\hline C & 0.91 & 0.92 & 0.81 \\
\hline G & 0.90 & 0.89 & 0.74 \\
\hline $\mathrm{T}$ & 0.89 & 0.95 & 0.73 \\
\hline
\end{tabular}

acid. In order to identify "rare" codons (which may be important in translational control), for each amino acid the codon frequency of each synonymous codon was divided by the codon frequency of the codon with the highest codon frequency. These ratios for all the codons for all the amino acids were then pooled and sorted. The rank order of all ratios $\leq 0.50$ was assigned, with the codon having the lowest ratio over all amino acids and all codons designated as " 1 " in the rank ordering.

\section{Computation of Rare and Frequent Codon Fractions}

For an examination of the relationship of rare codons, frequent codons, and information values, rare codons were defined as the 15 codons identified as rare in Table 5 , and frequent codons were defined as those with a value of 1.000 in Table 5, with the exception of the codons of methionine and tryptophan, which have only one codon. Fractions of rare and frequent codons were computed by dividing the number of rare or frequent codons, respectively, by the total number of codons in the sequence.

In mouse, the rare and frequent codon computations were based upon the tabulation of (Nakamura 1998; www. kazusa.or.jp/codon/cgi-bin/showcodon.cgi?species=Mus+ muscalus + [gbrod]). The results (not shown) were essentially identical to those for human (Table 5).

\section{Computation of Information (bits)}

The method developed by Schneider et al. (1986) to compute the information content of binding sites was adapted to compute the information of an individual protein-coding nucleotide sequence with respect to usage of synonymous codons. Very briefly, in the method developed by Schneider, multiple alignment of $\mathrm{N}$ sequences results in $\mathrm{n}$ aligned columns representing a protein binding site. For each column, the information value is computed. Finally, the overall information value for the binding site is computed by adding the information values for each of the $\mathrm{n}$ columns. The ability to perform this addition is not trivial; it is a direct consequence of the additivity property of information values (Hamming 1980; Tom Schneider, "Information Theory Primer with an Appendix on Logarithms," www.lecb.ncifcrf.gov/ toms/paper/primer). The additivity property requires that the information values be statistically independent, as is expected to be the case here.

There is a direct analogy between the method developed here for computation of the information of an individual protein-coding nucleotide sequence with respect to usage of synonymous codons, and for computation of the information of a protein (e.g., a transcription factor) binding site using multiple alignment of related sequences (Schneider et al. 1986): in the method developed here, each coding sequence (RefSeq) is the analogy of a binding site. Each of the (encoded) amino acids is the analogy of one column in the DNA multiple alignment. A synonymous codon is the analogy of $A, C, G$, or T in the DNA multiple alignment. Computation of the information value for a single (encoded) amino acid is the analogy of computing the information for a given column of the multiple alignment. Computation of the overall information value for a sequence by adding the information values for each of the (encoded) amino acids is the analogy of computing the overall information value for the binding site by summing the information values for each of the columns in the DNA alignment example.

This information measure for a given sequence $\left(\mathrm{R}_{\text {sequence }}\right)$ was computed as the difference between the overall uncertainty $\mathrm{H}_{\mathrm{g}}$ (with respect to a set of sequences of which the given sequence is a member; the subscript " $\mathrm{g}$ " is intended to indicate "global" or "genomic") and the uncertainty for the given sequence $\mathrm{H}_{\mathrm{s}}$ (the subscript " $\mathrm{s}$ " is intended to indicate a "single" sequence) after applying the "Approximate method" for small sample size as described in the Appendix of Schneider et al. (1986). The general form for uncertainty $\mathrm{H}$ is

$$
\text { uncertainty }=\mathrm{H}=\sum_{i=1}^{i=n_{\text {aa }}}\left(\sum_{j=1}^{j=n_{\text {syncod }(i)}} p_{i, j} \log _{2}\left(p_{i, j}\right)\right)
$$

where $\mathrm{n}_{\mathrm{aa}}$ is the effective number of amino acids (in this case equal to 23 as described below), $\mathrm{n}_{\text {syncod(i) }}$ is the number of synonymous codons for amino acid $i$, and $p_{i, j}$ is the probability of synonymous codon $j$ for amino acid $i$. For the overall uncertainty $\mathrm{H}_{\mathrm{g}}, \mathrm{p}_{\mathrm{i}, \mathrm{j}}$ is computed over the entire set of sequences for the given organism (it is important to emphasize that $p_{i, j}$ is the actually measured frequency distribution and NOT based upon a prior assumption of uniform distribution), whereas for each individual sequence, the computation of $p_{i, j}$ is restricted to the codons for that one sequence to give $\mathrm{H}_{\mathrm{s}}$. This measures the diversity of synonymous codon usage within the given sequence. The general form for information is

$$
\text { information }=\mathrm{R}_{\text {sequence }}=\mathrm{H}_{\mathrm{g}}-\mathrm{H}_{\mathrm{S}}
$$

This is an analogy to information by Shannon (Shannon 1948; Schneider et al. 1986).

The effective number of amino acids is 23 , since the codons for leucine, serine, and arginine were each divided into two disjoint sets such that only codon position 3 substitutions were counted as possibly being synonymous. This approach introduces a slight error, since in fact there are several instances where codon position 1 substitutions might be synonymous (two for leucine (CTA/TTA and CTG/TTG), zero for serine, and two for arginine (CGA/AGA and CGG/AGG)). This 
slight error is not expected to alter any of the conclusions here. There are no cases where position two substitutions might be synonymous.

\section{Fitting the Information Function Using a Theoretical Model}

For the simple case of two independent variables, the uncertainty versus probability curve has a maximum at $p=0.50$ (Hamming 1980; Tom Schneider, "Information Theory Primer with an Appendix on Logarithms," www.lecb.ncifcrf. gov/ toms/paper/primer), or, equivalently, the information versus probability curve has a minimum at $p=0.50$. In general, for $\mathrm{n}$ independent variables, the uncertainty achieves a maximum and the information achieves a minimum when the probabilities of all $\mathrm{n}$ variables are equal to one another (Hamming 1980), and therefore, since the $\mathrm{p}$ values must sum to unity, when each is equal to $1 / \mathrm{n}$. Conversely, given a scatter plot of information versus the probability of one variable, the effective number of variables can be estimated as

$$
\mathrm{n}_{\text {effective }}=1 / \mathrm{p}_{\min }
$$

where $n_{\text {effective }}$ is the effective number of variables and $p_{\min }$ is the value of $p$ at which the information achieves a minimum.

For the data examined here, $\mathrm{n}_{\text {effective }}$ is either 2 or 4 (see Results). In the instances where $\mathrm{n}_{\text {effective }}=2$, with the exception of a scale factor, there are no free parameters (that is, there are zero degrees of freedom) in fitting a theoretical curve to the scatter plot: one of the degrees of freedom is used up by the constraint that the two $p$ values must sum to unity, and the other degree of freedom is used up by the fact that one of the $\mathrm{p}$ values is used as the $\mathrm{x}$ coordinate in the scatter plot.

In the theoretical curve, $\mathrm{H}_{\mathrm{g}}$ should be attributed a value that results from making the weakest possible assumption. This assumption is that the overall uncertainty is as high as possible. This condition will be met if all $p$ values are equal. In this case, Equation 2 for $\mathrm{H}_{\mathrm{g}}$ is given as $\log 2\left(\mathrm{n}_{\text {effective }}\right)=$ $\log 2(2)=1$. Thus, the data in the scatter plot will be fit to the theoretical curve (according to Equations 2 and 3):

$$
\text { information }=\alpha\left\{1-\left\{-\left[\mathrm{p} \log _{2}(\mathrm{p})+(1-\mathrm{p}) \log _{2}(1-\mathrm{p})\right]\right\}\right\}
$$

where the scale factor $\alpha$ is obviously the only adjustable parameter.

In the instances where $\mathrm{n}_{\text {effective }}=4$, in addition to the scale factor, there are now two free parameters. As just described for $\mathrm{n}_{\text {effective }}=2, \mathrm{H}_{\mathrm{g}}$ is now given as $\log 2\left(\mathrm{n}_{\text {effective }}\right)=$ $\log 2(4)=2$. For the sake of clarity, assume that the four probability values are $p_{1}, p_{2}, p_{3}$, and $p_{4}$. Further assume that $p_{1}$ is to be used as the $\mathrm{x}$ axis and that $\mathrm{p}_{4}$ is constrained by the relationship $\mathrm{p}_{1}+\mathrm{p}_{2}+\mathrm{p}_{3}+\mathrm{p}_{4}=1$, so that $\mathrm{p}_{2}$ and $\mathrm{p}_{3}$ are the adjustable parameters (subject to the constraint that $\mathrm{p}_{2}+\mathrm{p}_{3} \leq$ $1-\mathrm{p}_{1}$ ). In practice, an alternative simpler approach to performing the fitting was used here: $\mathrm{p}_{2}, \mathrm{p}_{3}$, and $\mathrm{p}_{4}$ were each set to be equal to $\left(1-p_{1}\right) / 3$, so that the scale factor was the only adjustable parameter. This approach worked well, and it was neither justifiable nor useful to adjust additional free parameters. In this case, (after algebraic simplification) information was given by

$$
\text { information }=\alpha\left\{2-\left\{-\left[p \log _{2}(p)+(1-p) \log _{2}((1-p) / 3)\right]\right\}\right\}
$$

In Figure 2C, D, and E, only the data in Figure 2E matched a theoretical curve for information (Equation 6). Therefore, the value for $\alpha$ was determined by fitting to data in Figure 2E, and then this same value for $\alpha$ was used to generate the theoretical curves in Figure $2 \mathrm{C}$ and $\mathrm{D}$, in order to show the large deviation of the data in Figure $2 \mathrm{C}$ and $\mathrm{D}$ from the theoretical curve. This was also true for Figure 4B, C, and D.

\section{ACKNOWLEDGMENTS}

I would like to thank Paul Sharp, Tom Schneider, Lukas Wagner, Greg Schuler, Wojciech Makalowski, and John Spouge for helpful interactions, Donna Maglott for the identification of orthologous pairs of human and mouse RefSeqs, and Deanna Church for prepublication results of Spidey RefSeq/genome alignments.

The publication costs of this article were defrayed in part by payment of page charges. This article must therefore be hereby marked "advertisement" in accordance with 18 USC section 1734 solely to indicate this fact.

\section{REFERENCES}

Andersson, S.G., and Sharp, P.M. 1996. Codon usage and base composition in Rickettsia prowazekii. J. Mol. Evol. 42: 525-536.

Aota, S., and Ikemura, T. 1986. Diversity in G + C content at the third position of codons in vertebrate genes and its cause. Nucleic Acids Res. 14: 6345-6355.

Bernardi, G. 2000a. The compositional evolution of vertebrate genomes. Gene 259: 31-43.

Bernardi, G. 2000b. Isochores and the evolutionary genomics of vertebrates. Gene 241: 3-17.

Bulmer, M.A. 1987 Statistical analysis of nucleotide sequences of introns and exons in human genes. Mol. Biol. Evol. 4: 395-405.

Cruveiller, S., D'Onofrio, G., and Bernardi, G. 2000 The compositional transition between the genomes of cold- and warm-blooded vertebrates: Codon frequencies in orthologous genes. Gene 261: 71-83.

Filipski, J, Salinas, J., Rodier, F. 1987 Two distinct compositional classes of vertebrate gene-bearing DNA stretches, their structures and possible evolutionary origin. DNA 6: 109-118.

Grocock, R.J., and Sharp, P.M. 2001. Synonymous codon usage in Cryptosporidium parvum: Identification of two distinct trends among genes. Int. J. Parasitol. 31: 402-412.

Hamming, R.W. 1980. Entropy and Shannon's First Theorem. In Coding and information theory. pp. 107. Prentice-Hall Inc. Englewood Cliffs, New Jersey.

Ikemura, T. 1981. Correlation between the abundance of Escherichia coli transfer RNAs and the occurrence of the respective codons in its protein genes: A proposal for a synonymous codon choice that is optimal for the E. coli translational system. J. Mol. Biol. 151: 389-409.

Ikemura, T. 1985. Codon usage and tRNA content in unicellular and multicellular organisms. Mol. Biol. Evol. 2: 13-34.

Ikemura, T., and Aota, S. 1988. Global variation in G+C content along vertebrate genome DNA. Possible correlation with chromosome band structures. J. Mol. Biol. 203: 1-13.

Ikemura, T., Aota, S., Kawasaki, K., and Ozeki, H. 1983. Codon choice pattern of higher eukaryotes. Jpn. J. Genet. 58: 648.

Ikemura, T., and Wada, K. 1991. Evident diversity of codon usage patterns of human genes with respect to chromosome banding patterns and chromosome numbers; relation between nucleotide sequence data and cytogenetic data. Nucleic Acids Res. 19: 4333-4339.

Ikemura, T., Wada, K., and Aota, S. 1990. Giant G+C\% mosaic structures of the human genome found by arrangement of GenBank human DNA sequences according to genetic positions. Genomics 8: 207-216.

Karlin, S., and Mrázek, J. 1996. What drives codon choices in human genes? J. Mol. Biol. 262: 459-472.

Kimura, M. 1983. The neutral theory of molecular evolution. Cambridge University Press, Cambridge.

Kliman, R.M., and Hey, J. 1994 The effects of mutation and natural selection on codon bias in the genes of Drosophila. Genetics 137: 1049-1056.

Knight, R.D., Freeland, S.J., and Landweber, L.F. 2001. A simple model based on mutation and selection explains trends in codon and amino-acid usage and GC composition within and across genomes. Genome Biol. 2. 
Lafay, B., Atherton, J.C., and Sharp, P.M. 2000. Absence of translationally selected synonymous codon usage bias in Helicobacter pylori. Microbiology 146: 851-860.

Lafay, B., and Sharp, P.M. 1999 . Synonymous codon usage variation among Giardia lamblia genes and isolates. Mol. Biol. Evol. 16: $1484-1495$.

Li, W.-H. 1997. Rates and Patterns of Nucleotide Substitution. In Molecular evolution. pp. 196-202. Sinauer Associates, Sunderland MA.

Lloyd, A.T., and Sharp, P.M. 1993 . Evolution of the RecA gene and the molecular phylogeny of bacteria. J. Mol. Evol. 37: 399-407.

Makalowski, W., and Boguski, M.S. 1998. Evolutionary parameters of the transcribed mammalian genome: An analysis of 2,820 orthologous rodent and human sequences. Proc. Natl. Acad. Sci. 95: 9407-9412.

Marin, A., Bertranpetit, J., Oliver, J.L., and Medina, J.R. 1989. Variation in G + C-content and codon choice: Differences among synonymous codon groups in vertebrate genes. Nucleic Acids Res. 17: 6181-6189.

Mouchiroud, D., and Bernardi, G. 1993. Compositional properties of coding sequences and mammalian phylogeny. J. Mol. Evol. 37: 109-116.

Mouchiroud, D., Fichant, G., and Bernardi, G. 1987. Compositional compartmentalization and gene composition in the genome of vertebrates. J. Mol. Evol. 26: 198-204.

Muto, A., and Osawa, S. 1987. The guanine and cytosine content of genomic DNA and bacterial evolution. Proc. Natl. Acad. Sci. 84: $166-169$.

Nakamura, Y., Gojobori, T., and Ikemura, T. 1998. Codon usage tabulated from the International DNA Sequence Databases. Nucleic Acids Res. 26: 334.

Pruitt, K.D., Katz, K.S., Sicotte, H., and Maglott, D.R. 2000. Introducing RefSeq and LocusLink: Curated human genome resources at the NCBI. Trends Genet. 16: 44-47.

Pruitt, K.D., and Maglott, D.R. 2001. RefSeq and LocusLink: NCBI gene-centered resources. Nucleic Acids Res. 29: 137-140.

Schneider, T.D., Stormo, G.D., Gold, L., and Ehrenfeucht, A. 1986. Information content of binding sites on nucleotide sequences. $J$. Mol. Biol. 188: 415-431.
Shannon, C.E. 1948. A mathematical theory of communication. Bell System Tech. J. 27: 379-423, 623-656.

Sharp, P.M., Averof, M., Lloyd, A.T., Matassi, G., and Peden, J.F. 1995. DNA sequence evolution: The sounds of silence. Philos. Trans. R. Soc. Lond. B. Biol. Sci. 349: 241-247.

Sharp, P.M., Cowe, E., Higgins, D.G., Shields, D.C., Wolfe, K.H., and Wright, F. 1988. Codon usage patterns in Escherichia coli, Bacillus subtilis, Saccharomyces cerevisiae, Schizosaccharomyces pombe, Drosophila melanogaster and Homo sapiens: A review of the considerable within-species diversity. Nucleic Acids Res. 16: $8207-8211$

Sharp, P.M., and Li, W.H. 1986. An evolutionary perspective on synonymous codon usage in unicellular organisms. J. Mol. Evol. 24: $28-38$.

Sharp, P.M., and Matassi, G. 1994. Codon usage and genome evolution. Curr. Opin. Genet. Dev. 4: 851-860.

Sharp, P.M., Stenico, M., Peden, J.F., and Lloyd, A.T. 1993. Codon usage: Mutational bias, translational selection, or both? Biochem. Soc. Trans. 21: 835-841.

Stenico, M., Lloyd, A.T., and Sharp, P.M. 1994. Codon usage in Caenorhabditis elegans: Delineation of translational selection and mutational biases. Nucleic Acids Res. 22: 2437-2446.

Sueoka, N., and Kawanishi, Y. 2000. DNA G+C content of the third codon position and codon usage biases of human genes. Gene 261: 53-62.

Tao, X. and Dafu,D. 1998. The relationship between synonymous codon usage and protein structure. FEBS Lett. 434: 93-96

Wheelan, S.J., Church, D.M., and Ostell, J.M. 2001. Spidey: A tool for mRNA-to-genomic alignments. Genome Res. 11: 1952-1957.

\section{WEB SITE REFERENCES}

www.kazusa.or.jp/codon/cgi-bin/showcodon.cgi?species= Homo+sapiens+[gbpri]

www.lecb.ncifcrf.gov/ toms/paper/primer

www.ncbi.nlm.nih.gov

Received August 31, 2001; accepted in revised form March 6, 2002.
Genome Research www.genome.org 


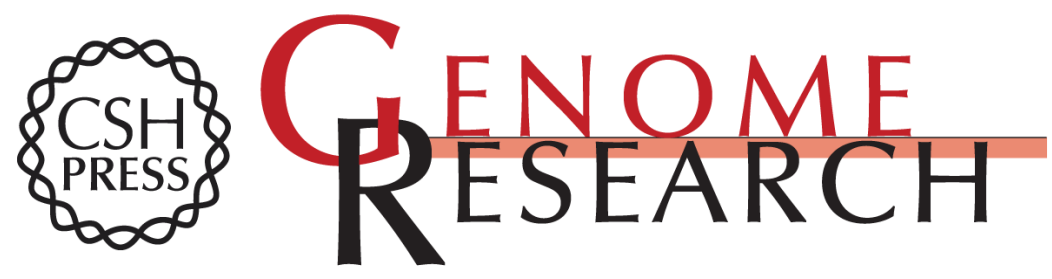

\section{Shannon Information Theoretic Computation of Synonymous Codon Usage Biases in Coding Regions of Human and Mouse Genomes}

\section{Barry Zeeberg}

Genome Res. 2002 12: 944-955

Access the most recent version at doi:10.1101/gr.213402

References This article cites 38 articles, 5 of which can be accessed free at: http://genome.cshlp.org/content/12/6/944.full.html\#ref-list-1

\section{License}

Email Alerting Service
Receive free email alerts when new articles cite this article - sign up in the box at the top right corner of the article or click here.

\section{Affordable, Accurate Sequencing.}

